INPLASY

PROTOCOL

To cite: Reyes et al.

Participatory approaches to empower girls and young women in physical education and sports contexts: a systematic review. Inplasy protocol 2021100026. doi: 10.37766/inplasy2021.10.0026

Received: 08 October 2021

Published: 08 October 2021

Corresponding author: Lucía Reyes

lucia.reyes@uclm.es

Author Affiliation: Universidad de Castilla-La Mancha, Facultad de Educación de Albacete.

Support: Ministry of Universities.

Review Stage at time of this submission: Formal screening of search results against eligibility criteria.

\section{Participatory approaches to empower girls and young women in physical education and sports contexts: a systematic review}

Reyes, L1; García López, LM2; Camacho-Miñano, MJ4; Oliver, $\mathrm{KL}^{4}$.
Review question / Objective: The purpose of this work is to systematically review the existing scientific literature on the use of participatory research approaches for the empowerment of girls and young women in physical education and sport contexts.

Condition being studied: The empowerment of girls and young women in sports-physical activity contexts by means of participatory approaches.

Information sources: To carry out this systematic review, a bibliographic search was carried out in eight electronic databases (Academic Search Ultimate, ERIC, MedLine, Psyclnfo, Scopus, Sociology Source Ultimate, Sport-Discus and Web of Science) between the months of April and May 2021.

INPLASY registration number: This protocol was registered with the International Platform of Registered Systematic Review and Meta-Analysis Protocols (INPLASY) on 08 October 2021 and was last updated on 08 October 2021 (registration number INPLASY2021100026).

Conflicts of interest:

None declared.

\section{INTRODUCTION}

Review question / Objective: The purpose of this work is to systematically review the existing scientific literature on the use of participatory research approaches for the empowerment of girls and young women in physical education and sport contexts.

Rationale: Despite the advances in last decades, recent studies confirm that gender stereotypes are still present in sports settings and in school physical 
education (PE) (Metcalfe, 2018; Roberts et al., 2020; Scraton, 2018). In this way, the field of physical activity and sports constitutes a strong factor of social influence since, in a traditional way, this environment has considered as inferior and has reacted negatively to the characteristics and behaviors associated with femininity (Frosh et al., 2002). That is why, due to the powerful influence of gender norms on masculinity and femininity, at present, the participation and experiences of girls in the contexts of PE and sport are still limited (Walseth et al., 2017). Consequently, due to the low participation data and their higher rates of abandonment of the practice, girls and adolescents have been considered as a target group who need of specific actions to promote physical activities and sports (Camacho-Miñano et al., 2011). The traditional idea that positions girls themselves as the culprits for their lack of involvement in $P E$ and sport is a misconception as it places responsibility on themselves, regardless of their social reality. For this reason, to help girls to cope with these types of situations, pedagogical approaches are necessary that prioritize their action on creating opportunities, taking into account the participants themselves and their learning process (Kirk, 2010). Participatory action research designs, and specifically the activist approach, coincide with this approach. These designs consider girls as participants who are actively involved in their learning process and do so by acquiring a role as co-investigators (Fisette, 2008; Hamzeh, 2007; Oliver et al., 2009). Within the particiaparory designs, the activist approach is a pedagogical model that includes in its own architecture the fundamental elements of a studentcentered pedagogy, action- research, and that considers listening to $t$ the students' voices to respond them (Oliver and Kirk, 2015). Consequently, from $P E$ and sport contexts, listening to the voice of girls and considering their concerns, it will be possible to create resources and learning experiences that contribute to their empowerment process as women.
Condition being studied: The empowerment of girls and young women in sports-physical activity contexts by means of participatory approaches.

\section{METHODS}

Search strategy: The terms searched in Spanish and English are: (School OR Physical Education OR Sport * OR Physical Activity OR Sports Club OR Exercise OR After-school sport OR Community programs) AND ("Positive youth development" * OR Empowerment * OR "Gender" * OR "Social Justice" OR "Social Transformation" OR Emancipation *) AND (Participatory * OR Activist * OR Community *) AND (Child * OR Adolescent * OR Student * OR Youth * OR Student * OR Girl *).

Participant or population: Girls and young women between the ages of six and 18 of any ethnicity.

Intervention: Intervention programs based on a participatory action-research design and developed with girls and young women in any context of physical-sporting activity.

Comparator: Purpose of the program, its duration, pedagogical strategies used.

Study designs to be included: The selected studies are based on participatory action research designs. These designs intend to help the members of a specific community or group to develop a critical awareness regarding the reality that surrounds them and thus begin their process of change. In this way, the participants acquire their own voice and a leading role throughout their learning process that has a positive impact on their feeling of belonging within the group and the research (Balcazar, 2003). To provide the participants with their own voice, it is essential to listen to their needs and interests, which will serve as a starting point to articulate intervention programs.

Eligibility criteria: Inclusion criteria: a) the participants were between six and 18 years old, b) the study had to be carried out in any physical activity context, c) the study 
design had to be based on participatory action research, d) the objective of the study had to be to empower girls in PE and sport contexts, e) studies published between 1990 and 2021 and f) published in Spanish or English.

Information sources: To carry out this systematic review, a bibliographic search was carried out in eight electronic databases (Academic Search Ultimate, ERIC, MedLine, Psyclnfo, Scopus, Sociology Source Ultimate, Sport-Discus and Web of Science) between the months of April and May 2021.

Main outcome(s): The results show the ability of these participatory approaches to develop critical awareness of girls in contexts of physical activity. In addition, they also allow us to know the benefits that the empowerment process of girls as women can have having a space where they can express their needs and concerns themselves.

Quality assessment / Risk of bias analysis: To evaluate the quality of the a priori studies, the Viswanathan \& Berkman (2012) tool will be used, selecting the most appropriate items. Depending on the characteristics of all the included studies, a more appropriate tool could be used.

Strategy of data synthesis: The analysis and interpretation of the results in this review was done in isolation with each of the studies based on common criteria to later draw the most relevant conclusions.

Subgroup analysis: Age, duration of the program, context of implementation, instruments for data collection.

Sensitivity analysis: The risk of bias will be explored using the Viswanathan \& Berkman (2012) tool.

Language: Spanish and English.

Country(ies) involved: Spain, United States.

Keywords: Participatory action research, activist approach, emancipation and youth.
Contributions of each author:

Author 1 - Lucía Reyes - Performed the search and screening, data synthesis and analysis, write and revise the manuscript.

Email: lucia.reyes@uclm.es

Author 2 - Luis Miguel García López Guided the search and the selection and analysis data, write and revise the manuscript.

Author 3 - María José Camacho-Miñano Guided the data analysis, write and revise the manuscript.

Author 4 - Kimberly Linda Oliver - Write and revise the manuscript. 